\title{
Temporal Modulation of Luminance Adapts Time Constant of Fly Movement Detectors
}

\author{
A. Borst and M. Egelhaaf \\ Max-Planck Institut für biologische Kybernetik, Spemannstrasse 38, D-7400 Tübingen, Federal Republic of Germany
}

\begin{abstract}
The time constant of movement detectors in the fly visual system has been proposed to adapt in response to moving stimuli (de Ruyter van Steveninck et al. 1986). The objective of the present study is to analyse, whether this adaptation can be induced as well, if the luminance of a stationary uniform field is modulated in time. The experiments were done on motion-sensitive wide-field neurones of the lobula plate, the posterior part of the third visual ganglion of the blowfly, Calliphora erythrocephala. These cells are assumed to receive input from large retinotopic arrays of movement detectors. In order to demonstrate that our results concern the properties of the movement detectors rather than those of a particular wide-field cell we recorded from two different types of them, the H1 - and the HSE-cell. Both cell types respond to a brief movement stimulus in their preferred direction with a transient excitation. This response decays about exponentially. The time constant of this decay reflects, in a first approximation, the time constant of the presynaptic movement detectors. It was determined after prestimulation of the cell by the following stimuli: $(a)$ periodic stationary grating; $(b)$ uniform field, the intensity of which was modulated sinusoidally in time (flicker stimulation); (c) periodic grating moving frontto-back; $(d)$ periodic grating moving back-to-front. The decay of the response is significantly faster not only after movement but also after flicker stimulation as compared with pre-stimulation with a stationary stimulus. This is interpreted as an adaptation of the movement detector's time constant. The finding that flicker stimulation also leads to an adaptation shows that movement is not necessary for this process. Instead the adaptation of the time constant appears to be governed mainly by the temporal modulation (i.e., contrast frequency) of the signal in each visual channel.
\end{abstract}

\section{Introduction}

Movement detection is one of the basic tasks the visual system has to perform. Much evidence has been accumulated in the last few decades that the underlying mechanism is based on a multiplication-like interaction of the signals coming from adjacent visual elements (Reichardt 1961): one of the signals is delayed by some kind of low-pass filter and is subsequently multiplied with the instantaneous signal of the neighbouring input channel. If such a "correlation-type" of movement detector is stimulated by a periodic grating moving with a constant velocity, it shows a response optimum at a certain contrast frequency, i.e., the number of spatial periods which pass a single visual element per second (e.g., Götz 1972). The optimum contrast frequency is independent of the spatial wavelength of the pattern and is determined by the time constant of the movement detector filter. Under transient stimulus conditions the filter time constant determines the dynamic range in which the movement detector is operative (Egelhaaf and Reichardt 1987). In the special case of a moving pattern that stops abruptly the filter time constant is reflected in the time course of the decay of the movement detector signal.

A similar method has recently been employed by de Ruyter van Steveninck et al. (1986) to estimate the movement detector time constant. They recorded from a directionally-selective motion-sensitive interneurone in the lobula plate, the posterior part of the third visual ganglion of the blowfly Calliphora erythrocephala. This cell, called $H 1$, is assumed to receive retinotopic input by local movement detectors from almost the entire visual field of one eye (see Hausen 1981). If the H1-cell is stimulated with a stepwise pattern displacement its average firing rate has been found to increase sharply 
and then to decay about exponentially to the cell's resting activity. The most important result of the study of de Ruyter van Steveninck et al. (1986) is the finding that the time constant of this exponential decay depends on the stimulus history. If the cell is exposed to pattern motion in either horizontal direction prior to the test step, the decay is significantly faster. This finding is interpreted as an adaptation of the movement detector time constant. It has been proposed by these authors that "the response time constant is set by angular velocity and not by contrast frequency" (de Ruyter van Steveninck et al. 1986, p. 228). To achieve this they further conclude that "the visual system can in effect estimate the local stimulus velocity" (ibid, p. 234). However, local velocity measurements, irrespective of the textural properties of the pattern, cannot be done by a correlation-type of movement detector (e.g. Reichardt 1961; Egelhaaf and Reichardt 1987). As a consequence, one should expect that the fly visual system comprises two parallel retinotopic arrays of movement detectors: One of them should be the wellestablished correlation-type of movement detector known for long to control the optomotor turning reaction of the fly (Götz 1965; Buchner 1984). It also provides the input to the lobula plate large-field neurones such as the H1-cell (e.g., Hausen 1981). The other type of movement detector should be a pure velocity sensor with the sole function to adapt the filter time constant of the other detector.

This scheme appears to us rather unplausible and will be challenged in the present paper by a simple qualitative experiment. It will be tested, whether the decay of the cell's response to a brief motion stimulus is shortened by pre-stimulation only with moving patterns or also if the luminance of a stationary uniform field is modulated in time ("flicker"). Since the flicker stimulation contains no movement and, therefore, no velocity information at all, adaptation to it would be difficult to reconcile with the view of the de Ruyter van Steveninck et al. (1986) that the adaptation of the movement detector time constant is governed by pattern velocity. Instead, it would speak in favour of the interpretation that it is controlled by the temporal modulation, i.e. the contrast frequency, in each visual channel. The experiments were performed not only on the H1-cell but also on another lobula plate large-field neurone, the HSE-cell (see Hausen 1982a, b).

\section{Materials and Methods}

Animals. A1l experiments were performed on female Calliphora erythrocephala which were between 1 and 3 days old. They were immobilzed, had their legs cut and were glued with their back to a small piece of glas. For insertion of the electrode into the lobula-plate their head capsule was opened from behind (for details see Hausen 1982a).

Electrodes. Intracellular electrodes were pulled on a micropipette puller (Brown and Flaming) using capillaries of $1 \mathrm{~mm}$ diameter (Clark Electromedical Instruments). They were filled with $\mathrm{KCl}$ and had a resistance of about $50 \mathrm{M} \Omega$. For extracellular recording we used tungsten electrodes the tips of which were sharpened electrolytically. They were insulated with varnish (Insl- $X$ ) and had a resistance of about $5 \mathrm{M} \Omega$ (see Hausen 1982a). In all experiments the indifferent electrode was a glass capillary filled with ringer solution and inserted into the hemolymph of the head capsule.

Experimental Set-Up. Flies were mounted on a holder facing a monitor (Tektronix 602) of $81^{\circ}$ vertical and $69^{\circ}$ horizontal extent with respect to the fly. On this monitor various patterns were displayed by an image synthesizer ("Picasso", Innisfree). The grating had a spatial wavelength of $\lambda=23^{\circ}$ as seen by the fly in the middle of the screen and a contrast $C=0.6$.

Experimental Procedure. After successful penetration of a HSE-cell or sufficient signal-to-noise ratio of the H1-cell the stimuli were given in the following temporal order: $3 \mathrm{~s}$ adapting stimulus, $200 \mathrm{~ms}$ stationary pattern, $20 \mathrm{~ms}$ test stimulus and $3 \mathrm{~s}$ stationary pattern. These cycles were repeated 20 times in a sequence for each of five different adapting stimuli (grating moving from front-to-back, grating moving from back-to-front, grating at rest, flicker, stationary uniform field). As an adapting stimulus the grating was moved with a contrast frequency of $c f=4 \mathrm{~Hz}$. For the test stimulus it was moved in the cell's preferred direction (H1: back-to-front; HSE front-to-back) for $20 \mathrm{~ms}$ with a contrast frequency of $c f=6 \mathrm{~Hz}$. When flicker was given as an adapting stimulus the overall light intensity was modulated sinusoidally with the same contrast and the same contrast frequency as above $(C=0.6, c f=4 \mathrm{~Hz})$. Experiments were repeated as long as a stable recording was possible, resulting in up to 160 sweeps per adapting stimulus for the H1-cell and up to 60 sweeps per adapting stimulus for the HSE-cell. The duration, contrast and contrast frequency of the adapting stimuli were chosen as to result in an intermediate state of adaptation: The decay of the response to a brief motion step was significantly shorter than in the unadapted state, but still long enough so that, in principle, it could have been further reduced.

Data Evaluation. Extracellular signals were fed through a threshold discriminator. The signals were recorded on a tape recorder. Intracellular graded potentials were directly recorded. The data were replayed to a signal averager (Princeton, Mod. 4202) and subsequently processed by an IBM PC. 


\section{Results}

We recorded the response of two types of movement sensitive interneurones to a brief movement stimulus ("test stimulus") after various preceding stimuli ("adapting stimuli"). Both the H1-cell and the HSEneurone reside in the lobula plate, the posterior part of the third visual ganglion of the fly's brain. They receive input from large parts of the visual field and respond selectively to the direction of motion. The H1-cell is excited by back-to-front, the HSE-neurone by frontto-back motion. They are inhibited by motion in the respective opposite directions (see Hausen 1981). Both cell types differ in their characteristic response mode: While the H1-cell responds with a regular train of overshooting action potentials to excitation, the main response mode of the HSE-neurone are graded changes of the membrane potential which may be superimposed by small-amplitude spike-like potentials (see Hausen 1981, 1982a).

The response of the H1-cell to a $20 \mathrm{~ms}$ movement stimulus in the cell's preferred direction is characterized by a sharp increase in the firing rate; the HSE-cell is quickly depolarized under these stimulus conditions. This transient excitation is followed in both cell types by a slower approximately exponential decay of the response to the cell's resting activity. This is illustrated in the diagrams of Fig. 1 which show a spike frequency histogram of the H1-cell response to a number of stimulus presentations as well as the averaged graded membrane potential changes of a HSE-cell.
The time constant of the exponential decay of the response is likely to reflect the filter time constant of the movement detectors which are presynaptic to the H1and the HSE-cell (de Ruyter van Steveninck et al. 1986). To estimate the time constant from the experimental data appropriately, it should be noted that the response profiles of both cell types usually have a double-peaked appearance in their initial phase. It can be shown by comparing the on-line data with the response averages that the first response peak is due to highly synchronized spikes at the onset of movement stimulation. The resulting pronounced response maximum thus does not reflect the signal amplitude of the presynaptic movement detectors which we wanted to study. It, therefore, was discarded in the further analysis and not taken into account for the estimation of the time constant. Since we wanted to argue only qualitatively, we used the time after which the signal had dropped to $36 \%(1 / e)$ of its amplitude as a crude but convenient estimate of the time constant (see Fig. 1).

In which way the time constant of the decay of the response to the test stimulus is affected by different adapting stimuli was analysed on the basis of data obtained from 5 H1- and 3 HSE-cells. An example of the responses under the different stimulus conditions is given in Fig. 2. The particular adapting stimuli are indicated in the figure. Since not only the time constant of the decay but also the amplitude of the test response is affected to some extent by the adapting stimuli (see calibration bars in Fig. 2), the response amplitudes

\section{H1 CELL}

HSE CELL

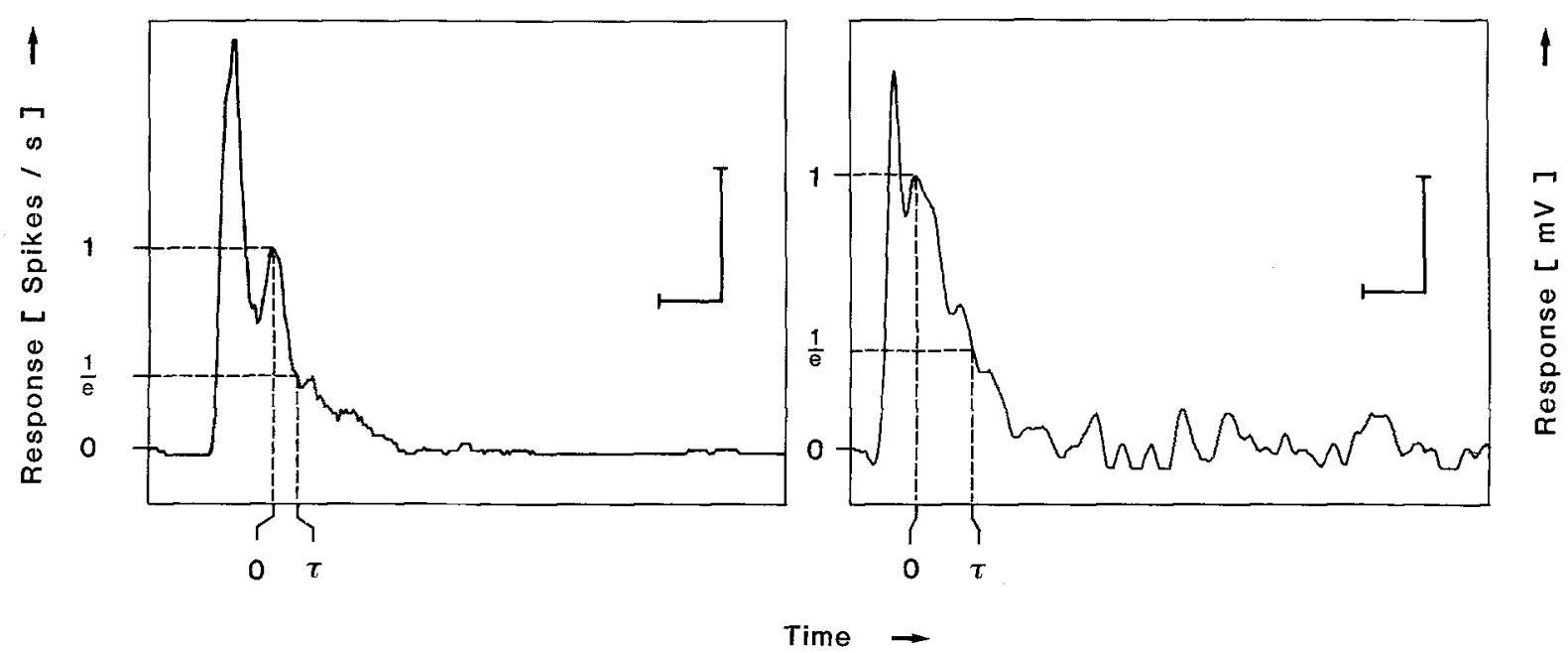

Fig. 1. Responses of large-field motion sensitive interneurones to a movement stimulus of $20 \mathrm{~ms}$ duration in the cell's preferred direction. The responses were averaged from several consecutive presentation of the stimulus program (140 H1; $32 \mathrm{HSE})$. Both the spike frequency histogram of the $\mathrm{H} 1$-cell and the graded membrane potential changes of the HSE-neurone have a double-peaked appearance. The initial peak is due to a synchronized spike and was discarded from the further analysis of the time constant $\tau$. Calibration bars: horizontal: $100 \mathrm{~ms}$; vertical: 50 spikes/s (H1), $5 \mathrm{mV}$ (HSE). For further details see text 
H1 CELL

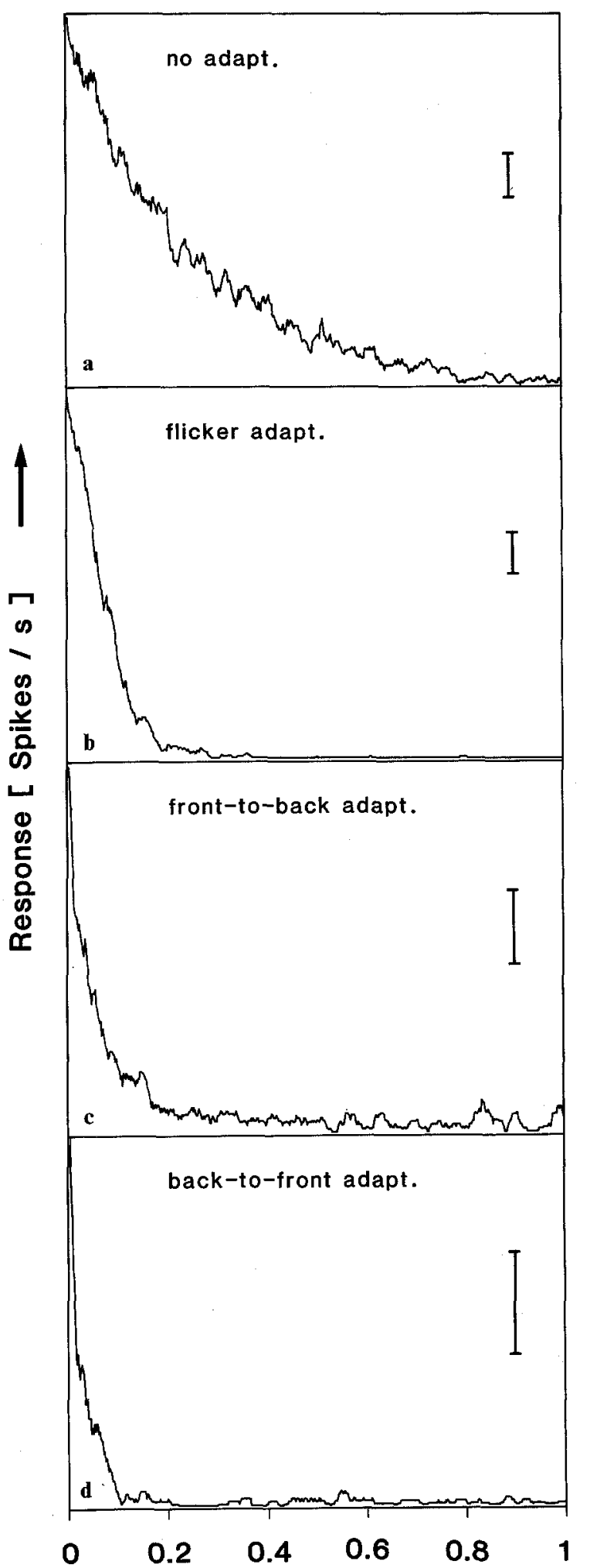

HSE CELL

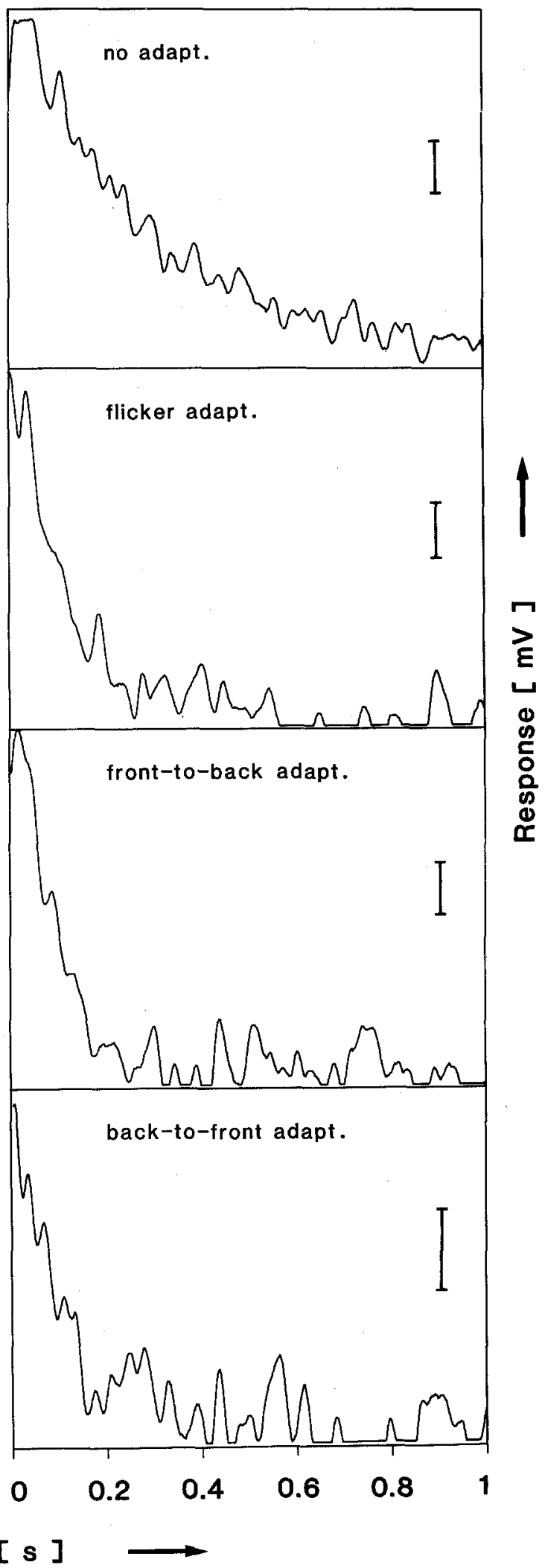


were normalized and their maximum value set to 1 . This facilitates a direct qualitative comparison of the experimental results. The influence of the adapting stimuli on the amplitude of the test response is of no immediate importance for the conclusions drawn in the present study and deserves further investigation. The close similarity of our results obtained from the H1- and the HSE-cell shows that $i$ ) the extracellularly recorded spike frequency of the H1-neurone indeed reflects the summated postsynaptic potentials of the cell, and that $i$ ) our interpretations on the nature of the adaptation process of the movement detector time constant are likely to be general ones rather than peculiar features of a particular cell type. The following statements refer to the averaged data of our experiments (see Fig. 2):

(1) Without preceding movement stimulation the response to the test stimulus decays from maximum response to the $36 \%$ level in about $288 \mathrm{~ms}$ in the $\mathrm{H} 1$ cells and $358 \mathrm{~ms}$ in the HSE-cells.

(2) With a grating moving from front-to-back as adapting stimulus the $36 \%$ level was reached after an average time intervall of $84 \mathrm{~ms}$ (H1-cell) and $108 \mathrm{~ms}$ (HSE-cell). This corresponds to an average shortening of the filter time constant in both cells to $30 \%$ of the unadapted state [compare with (1)].

(3) When the grating was moving from back-tofront during the adaptation period, the response to the test stimulus decayed to the $36 \%$ level within $63 \mathrm{~ms}$ (H1-cell) and $98 \mathrm{~ms}$ (HSE-cell) respectively, which is $22 \%$ (H1-cell) or $27 \%$ (HSE-cell) of the time constant without adaptation [compare with (1)].

(4) After flicker adaptation the response to the test stimulus reached the $36 \%$ level after $105 \mathrm{~ms}$ (H1cell) and $148 \mathrm{~ms}$ (HSE-celi). This corresponds to 38\% and $39 \%$ of the time constant in the unadapted state, respectively [compare with (1)]. This shows that the adaptation of the time constant after flicker stimulation is strong and highly significant, but on the average, though not in all flies, slightly less than after movement stimulation [compare with (2) and (3)]. Before these conclusions could be accepted a control experiment was done. This was necessary since after flicker adaptation the uniform screen had to be changed to a grating which subsequently formed the test stimulus. This switching might, in principle, lead to apparent motion which in turn might decrease the time constant. It was tested whether switching the pattern screen from a uniform field to a grating without preceding flicker had an adapting effect on its own (data not shown). The time constant in this case was reduced to about $87 \%$ of the value found without adaptation [see (1)]. It can thus be concluded that the decrease of the time constant observed after flicker adaptation is mainly due to the sinusoidal intensity modulation of the screen. Hence, movement information is no requirement for a decrease of the time constant characterizing the decay of the response to a brief motion stimulus.

\section{Discussion}

On the basis of our present experiments we have drawn the conclusion that intensity modulation of a stationary, uniform field leads to an adaptation of the time constant of the movement detector filters. Hence, movement does not represent a necessary condition for this kind of adaptation to occur. This conclusion needs some further qualification and has certain implications on the nature of the adaptation process which will be discussed in the following.

\section{Determination of the Time Constant}

The response of the recorded movement-sensitive wide-field cells of the lobula plate to a brief movement stimulus was found to decay about exponentially. The time constant of this exponential decay has been interpreted to reflect the time constant of the local movement detectors which are assumed to be presynaptic to the wide-field neurones (see also de Ruyter van Steveninck et al. 1986). This conclusion is correct as long as the only elements in the visual pathway between the retina and the lobula plate cells which have a time constant are the movement detector filters. It is unlikely that the adaptation process takes place after spatial integration of the local movement information. Instead, it has been shown to be essentially due to local filter properties of the visual system (de Ruyter van Steveninck et al. 1986). The possibility that the time course of the movement detector output is affected by processing of the incoming signals distally to the movement detectors has to be taken more seriously, although there is no evidence on the basis of our experimental data in favour of such a scheme. It can be easily shown by computer simulations that peripheral pre-filtering of the signals to both movement detector input channels can considerably affect the

Fig. 2a-d. Responses of the H1-cell and the HSE-cell to a $20 \mathrm{~ms}$ pulse of pattern movement in the cell's preferred direction after preexposure to four different adapting stimuli (from top to bottom): a grating not moving, b flicker stimulation, $\mathbf{c}$ grating moving front-toback, d grating moving back-to-front. Note that after flicker stimulation the response decays almost as fast as after movement of the grating. Data are averages of 140 (H1-cell) and 32 (HSE-cell) sweeps per adapting stimulus. Calibration bars: 20 spikes/s (H1); 2 mV (HSE) 
time course of the detector response to a brief movement step. With a peripheral low-pass filter the time constant of the decay of the test response is the more prolonged the larger the time constant of the peripheral filter as compared with the time constant of the movement detector. On the other hand, if the peripheral time constant is much smaller than the detector time constant it has only little influence in determining the time course of the response. This problem cannot be settled on the basis of only the exponential decay of the response to a brief movement stimulus. Nevertheless, there are two reasons that might speak in favour of the conclusion that the time course of the test response is essentially determined by the movement detector filter rather than by more peripheral pre-filtering.

i) It has been concluded in a recent study on the dynamic response properties of movement detectors that the detector input function does not allow much pre-filtering. Otherwise the movement detector output would be in contrast to the experimental results on its characteristic dynamic response properties (Egelhaaf and Reichardt 1987).

ii) Although there is an extensive transformation of the retinal light intensity distribution in the peripheral visual system in the fly, its role in the context of movement detection has by no means been settled so far. It is only clear, for obvious reasons, that the photoreceptors which can be regarded as some kind of temporal low-pass filters are presynaptic to the movement detectors. Although no simple low-pass filter can account for the temporal filter properties of the photoreceptors, their impulse-response in the light adapted state is significantly briefer than even the smallest time constants estimated for the movement detector filters (compare e.g., Howard et al. 1984 with de Ruyter van Steveninck et al. 1986; Egelhaaf and Reichardt 1987). In our experiments the adapting stimuli were chosen so that the resulting time constants of decay of the test response were much longer. Hence, we concluded that under our experimental conditions these time constants are not much affected by temporal filtering taking place in the retina and that the time constant of the decay of the response to a brief movement stimulus is essentially determined by the movement detector time constant.

\section{Stimulus Parameters Governing the Adaptation of the Time Constant}

The response to a brief movement step in the cell's preferred direction has been found to narrow considerably, if the animal was preexposed to large-field motion (see Fig. 2; Maddess and Laughlin 1985; de Ruyter van Steveninck et al. 1986). This change in the time course of the response has been interpreted as an adaptation of the movement detector time constant (de Ruyter van Steveninck et al. 1986). The strength of adaptation was found by these authors to be independent of the direction of motion. This can neither be confirmed nor excluded on the basis of our present data. Although under our stimulus conditions we found a difference in the time constant after adaptation to front-to-back and back-to-front motion respectively, this difference might be too small to allow a definite statement in this respect.

The main objective of the present study was to show that the movement detector time constant is reduced almost as much by sinusoidally modulating the luminance of a spatially uniform screen as after movement stimulation with the same contrast frequency. Hence, movement information is not required for an adaptation of the movement detector time constant. The fact that there is no velocity information at all in our flicker stimulus clearly excludes the hypothesis of de Ruyter van Steveninck et al. (1986) that the adaptation of the time constant is essentially governed by the velocity of the stimulus pattern irrespective of its spatial frequency content. This evidence appears to us convincing, since we decide between the two alternatives on the basis of a qualitative experiment. In contrast, de Ruyter van Steveninck et al. (1986) rely solely on quantitative statistical reasoning. Moreover, there are two findings of de Ruyter van Steveninck et al. (1986) which are predicted by our hypothesis but are not accounted for by these authors. $i$ ) The adaptation depends on pattern contrast. ii) The range of adaptation is limited by contrast frequency rather than by velocity. All this evidence is in favour of the hypothesis that the adaptational state of the movement detector is governed mainly by the temporal modulation, i.e. the contrast frequency, of the signal in the movement detector input channels. A minor discrepancy in the strength of adaptation after movement and flicker stimulation suggests that a certain degree of adaptation is likely to take place in the movement detectors after the multiplication-like interaction of the neighbouring channels. The major component of adaptation of the detector time constant, however, is determined by the frequency of modulation and the amplitude of the signals before they are multiplied.

\section{Consequences}

Our finding that the adaptation of the filter time constant is mainly governed by contrast frequency has certain implications for the operating range of the movement detection system. If the system is stimulated for a certain time with high contrast frequencies, the adaptation is strong and leads to a small time constant. This corresponds to a response optimum at high 
contrast frequencies. Low contrast frequencies result in a weak adaptation and, therefore, a large time constant. The system then has its optimum response at low contrast frequencies. By this mechanism, the otherwise relatively small range of contrast frequencies which result in large response amplitudes at the detector output is considerably increased. Broad contrast frequency optima have been found for the optomotor turning reaction (Götz 1965; Borst and Bahde 1987) and the response of lobula plate widefield neurones (Hausen 1982b). Since under low contrast conditions the adaptation is less pronounced (de Ruyter van Steveninck et al. 1986) the corresponding optimum range of contrast frequencies should be smaller and shifted towards lower values. Whether this prediction turns out to be true is currently being investigated (Borst and Bahde in prep.). Apart from the increased operating range of the movement detector, another advantage of an adjustable time constant is, that adaptation to short time constants is particularly important for a faithful representation of fast transient movement stimuli at the output of the motion detection system (Egelhaaf and Reichardt 1987). The flexibility of the time constant, therefore, allows the movement detection system to adapt to various environmental needs.

Acknowledgements. We are grateful to Prof. K.G. Götz, Dr. K. Hausen and Prof. W. Reichardt for stimulating discussions and for helpful criticism on the manuscript. It is a great pleasure to thank Susanne Bahde for her invaluable support in many respects. In particular, she provided us with excellent electrodes and skillfully prepared the figures.

\section{References}

Borst A, Bahde S (1987) Comparison between the movement detection systems underlying the optomotor and the landing response in flies. Biol Cybern 56:217
Buchner E (1984) Behavioural analysis of spatial vision in insects. In: Ali MA (ed) Photoreception and vision in invertebrates. Plenum Press, New York London, pp 561-621

Egelhaaf M, Reichardt W (1987) Dynamic response properties of movement detectors: Theoretical analysis and electrophysiological investigation in the visual system of the fly. Biol Cybern (in press)

Götz KG (1965) Behavioral analysis of the visual system of the fruitfly Drososphila. Proceedings of the symposium on information processing in sight sensory systems. Cal Tech, California Pasadena, pp 85-100

Götz KG (1972) Principles of optomotor reactions in insects. Bibl Ophthal 82:251-259

Hausen K (1981) Monocular and binocular computation of motion in the lobula plate of the fly. Verh Dtsch Zool Ges $74: 49-70$

Hausen K (1982a) Motion sensitive interneurons in the optomotor system of the fly. I. The horizontal cells: structure and signals. Biol Cybern 46:67-79

Hausen K (1982b) Motion sensitive interneurons in the optomotor system of the fly. II. The horizontal cells: receptive field organization and response characteristics. Biol Cybern 45:143-156

Howard J, Dubs A, Payne R (1984) The dynamics of phototransduction in insects. J Comp Physiol 154:707-718

Maddess T, Laughlin SB (1985) Adaptation of the motionsensitive neuron H1 is generated locally and governed by contrast frequency. Proc R Soc London Ser B 225:251-275

Reichardt W (1961) Autocorrelation, a principle for the evaluation of sensory information by the central nervous system. In: Rosenblith WA (ed) Sensory communication. MIT Press and Wiley, New York London, pp 303-317

Ruyter van Steveninck RR de, Zaagman WH, Mastebroek HAK (1986) Adaptation of transient responses of a movementsensitive neuron in the visual system of the blowfly Calliphora erythrocephala. Biol Cybern 53:451-463

Received: January 12, 1987

Dr. A. Borst

Dr. M. Egelhaaf

Max-Planck-Institut für Biologische Kybernetik

Spemannstrasse 38

D-7400 Tübingen

Federal Republic of Germany 